\title{
A study on estimation of 3-dimensional surface roughness of boring processes including kinematic motion deviations
}

\author{
Wiroj THASANA*, Nobuhiro SUGIMURA*, Koji IWAMURA* and Yoshitaka TANIMIZU* \\ *Mechanical Engineering, Graduate School of Engineering, Osaka Prefecture University \\ 1-1 Gakuen-cho, Naka-ku, Sakai-shi, Osaka 599.8531, Japan \\ E-mail: wiroj@tni.ac.th
}

Received 28 February 2014

\begin{abstract}
The surface roughness is one of the most important characteristics of machined parts. A systematic method is proposed in the paper to simulate the shape generation processes in the boring operations and to estimate the surface roughness of the generated faces, based on the machining parameters. The simulation model includes both the models of the shape generation motions considering kinematic motion deviations and the cutting tool geometries. The shape generation motions with deviations are mathematically described by 4 by 4 transformation matrices. A set of points on the bored holes are generated through the simulations, and an assessment surface is obtained as the datum reference to estimate the 2-dimensional (2D) and the 3-dimensional (3D) surface roughness, based on the points generated by the boring process simulations. The proposed method provides us with a systematic method to estimate the surface roughness in the boring processes including the kinematic motion deviations.
\end{abstract}

Key words : 3D surface roughness, Kinematic motion deviation, Boring processes, Shape generation motions, Machining process simulations

\section{Introduction}

In present global competitions, machine industries are constantly exploring systematic methods to increase the quality and the reliability of their products and to decrease the annual expenditures on the machining operations. Machining processes are inherently complex, and lead to use empirical methods for process developments. In particular, process parameters such as machining speeds, feed rates and tooling are usually selected based on handbooks and trial-and-error prototyping. However, these methods do not guarantee the process parameters which satisfy the required quality.

Since the early 1990s, a paradigm shift in manufacturing from 'real' to 'virtual' production has resulted in a build-up of research interests in the simulation techniques. With the aid of computers, it becomes possible to simulate some of the activities of physical manufacturing systems. The main objective of the virtual productions is to understand and to emulate the behavior of the manufacturing systems on the computers prior to the physical productions, aiming at reducing the amount of testing and experiments on the shop floors. They are so called as virtual manufacturing, virtual machine tools, virtual machining, virtual assembly, virtual tooling and virtual prototyping (Abdul et al. 2011). Some simulation models for the machining processes have recently been proposed to predict the dimensions, geometries and geometric deviations of the machined parts, aiming at investigating the suitable machining process parameters (Govik et al. 2012; Luo et al. 2010). However, the proposed models have been applied only to the limited areas of the machining processes and have not yet considered the verification of the generated faces.

One of the most important revolutions in the later part of the last century is introduction of CNC machine tools, which are able to carry out various complicated machining processes without human interaction. Various types of CNC machine tools are now being designed and applied to machining processes of complicated machine products. The machining accuracy and surface roughness is one of the most important characteristics of the CNC machine tools for generating the products with the high accuracy and the complicated geometries. 
Some researches have been carried out for the investigation of the machining processes based on the kinematic motion deviations between the tools and the workpieces (Sugimura et al. 2012). Li et al. (2008) conducted a survey of the off-line optimization on CNC machining based on the virtual machining and also discussed about the machining error compensation. This research did not consider the machining process analysis.

Atabey et al. (2003) have proposed a model for the analysis of boring process mechanics, which provides a method to estimate the chip thickness distributions along the cutting edges, based on the tool inclination angles, nose radius, depth of cut and feed rate. The three dimensional cutting forces are also estimated for the cases of the insert boring heads with run-out. Kaymakci et al. (2012) have proposed a unified cutting force model for turning, boring, drilling and milling operations with the inserted tools. The inserted tool tips and their orientations to the reference tool coordinates are mathematically represented by ISO tool definition standards. The friction forces acting on the rake faces are transformed into reference tool coordinates using the general transformation matrix.

However, the proposed model did not deal with how the individual models are integrated to estimate the final geometries of the machined parts, the geometric deviations and the surface roughness in the virtual machining processes.

As regards the surface roughness, Quinsat et al. (2008) have proposed a surface topography in ball end milling process by describing of 3D surface roughness parameters. The model has focused on free-form surface machining in order to produce 3D surface roughness parameters. Buj-Corral et al. (2012) have proposed a surface topography model in ball-end milling process as a function of feed per tooth and radial depth of cut. The numerical model was developed to predict topography and surface roughness based on geometric tool-workpiece intersection. It allows determining surface topography as a function of feed per tooth and revolution and radial depth of cut. However, the proposed model did not consider the kinematic motion deviations of boring processes.

The objective of the present research is to propose simulation model of the machine tools and the machining processes. In the present paper, a simulation model is proposed to simulate the boring process with kinematic motion deviations of the spindles. A set of points on the bored faces are obtained through the simulation. The model proposed represents the boring processes based on both the shape generation motions and the cutting tool geometries. The individual motions are mathematically described by 4 by 4 transformation matrices including the kinematic motion deviations. Emphasis is given to the modeling and analysis of the boring process of the single point tools. A systematic method is also proposed to verify the three-dimensional surface roughness of the bored faces base on the simulation results.

\section{Boring Process Simulation}

\subsection{Kinematic motions in boring processes}

The boring processes on CNC machining centers are carried out by both the spindle rotations and the linear feed motion along Z-axis, therefore, the motion deviations of the spindle rotation is an important issue for evaluating the geometric deviations of bored holes.

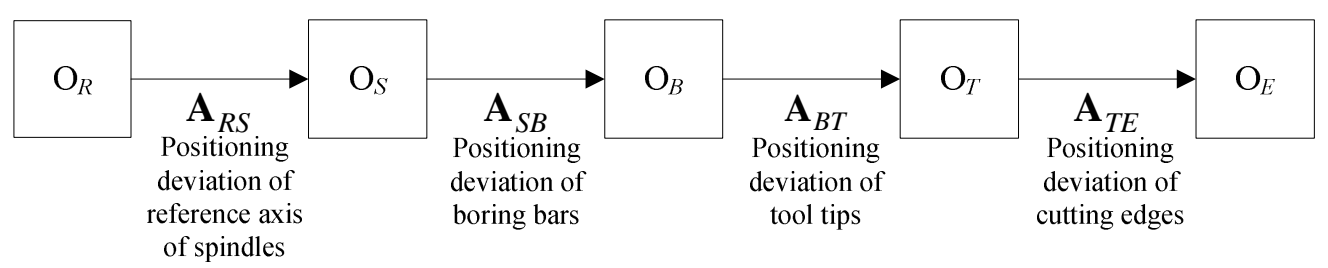

Fig. 1 Coordinate systems and their deviations

Five cartesian coordinate systems shown in Fig. 1 are set to represent the kinematic motion deviations. They are, $O_{R}, O_{S}, O_{B}, O_{T}$ and $O_{E}$ which represent the coordinate systems of the reference, spindles, boring bars, tool tips and cutting edges, respectively.

The shape generation processes of the machine tools are generally represented by the shape generation motions, and also the tool geometries. The shape generation motions, which are the relative motions of the tools against the 
workpieces, are executed by a set of rigid component of the machine tools (Sugimura and Murabe, 1997). The shape generation motions of the cutting edge against the reference are described by Eq. (1).

$\mathbf{X}_{R}=\mathbf{A}_{R S} \mathbf{A}_{S B} \mathbf{A}_{B T} \mathbf{A}_{T E} \mathbf{X}_{E}$

where,

$\mathbf{X}_{E}$ : Position vector of a point on the cutting edge in the cutting edge coordinate system.

$\mathbf{X}_{R}$ : Position vector of the point on the cutting edge in the reference coordinate system.

$\mathbf{A}_{i j}: 4$ by 4 homogeneous transformation matrices representing the relative positions and the kinematic motions between pairs of rigid bodies

The individual matrices include some kinematic and position deviations due to both the motion errors and the set-up errors, therefore, the kinematic motion deviations in the boring processes are discussed in the following section.

\subsection{Coordinate systems and deviation parameters}

The coordinate systems for the boring processes are shown in Fig. 2. The reference coordinate system $O_{R}$ is set according to ISO (International Standardization Organization) 230-1 (ISO 230-1: 2012, 2012; Choi et al. 2003), and the other coordinate systems are as follows.

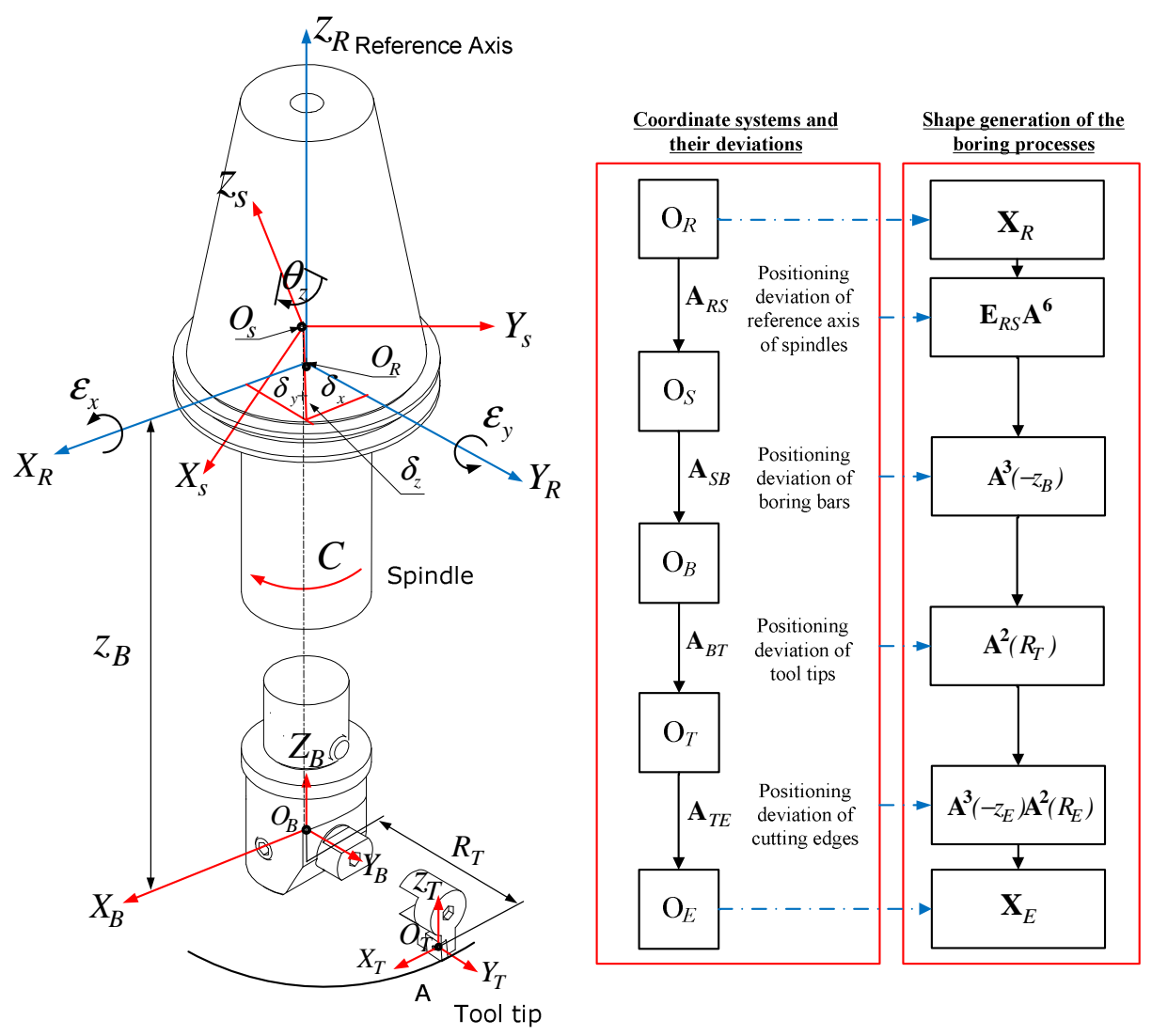

Fig. 2 Coordinate systems for boring processes

(1) The coordinate system $O_{S}$ of the spindle is fixed on the spindle and has four deviation parameters representing the position and orientation deviations of the average rotational axis of the spindle. They are $\delta_{x}(C), \delta_{y}(C), \varepsilon_{x}$ and $\varepsilon_{y}$ which represent the positioning deviations in $\mathrm{X}$ - and $\mathrm{Y}$-axis and the rotational deviations around $\mathrm{X}$ - and $\mathrm{Y}$-axis.

(2) The coordinate system $O_{B}$ of the boring bar is fixed on the boring bar and the parameter $z_{B}$ gives the distance between $O_{s}$ and $O_{B}$ representing the length of the boring bar. 
(3) The coordinate system $O_{T}$ of the tool tip is fixed on the tool tip and the parameter $R_{T}$ gives the distance between $O_{B}$ and $O_{T}$ representing the radial position of the tool tip against the boring bar axis.

(4) The coordinate system $O_{E}$ of the cutting edge is fixed on the cutting edge and the parameters $R_{E}$ and $z_{E}$ gives the distance between $O_{T}$ and $O_{E}$ representing the shapes and the dimensions of the tool tip.

The following equation is obtained from Eq. (1) by applying the parameters mentioned above.

$\mathbf{X}_{R}=\mathbf{E}_{R S} \mathbf{A}^{6}\left(\theta_{z}\right) \mathbf{A}^{3}\left(-z_{B}\right) \mathbf{A}^{2}\left(R_{T}\right) \mathbf{A}^{3}\left(-z_{E}\right) \mathbf{A}^{2}\left(R_{E}\right) \mathbf{X}_{E}$

where,

$\theta_{z} \quad$ : Rotational angle of spindle

$z_{B} \quad:$ Length along the Z-axis of the boring bar

$R_{T} \quad$ : Radial position of tool tip against the boring bar axis

$z_{E} \quad:$ Distance between $O_{T}$ and $O_{E}$ of the tool tip along the Z-axis

$R_{E} \quad:$ Distance between $O_{T}$ and $O_{E}$ of the tool tip along the Y-axis

$\mathbf{E}_{R S} \quad$ : Geometric deviations of spindle against reference coordinate system, which include all the deviation parameters, are given by the following equation.

$\mathbf{E}_{R S}=\left[\begin{array}{cccc}1 & 0 & \varepsilon_{y} & \delta_{x}(C) \\ 0 & 1 & -\varepsilon_{x} & \delta_{y}(C) \\ -\varepsilon_{y} & \varepsilon_{x} & 1 & 0 \\ 0 & 0 & 0 & 1\end{array}\right]$

where,

$\varepsilon_{x} \quad:$ Rotational deviation around $\mathrm{X}$-axis

$\varepsilon_{y} \quad:$ Rotational deviation around Y-axis

$\delta_{x}(C) \quad$ : Positioning deviation in $\mathrm{X}$-axis

$\delta_{y}(C) \quad$ : Positioning deviation in Y-axis

\subsection{Kinematic motion of cutting edge}

There are various inserted tool tip geometries are used in the cutting tools, and seventeen inserted tool tip shapes are defined in ISO 13399 standards (Kaymakci et al. 2012). The generalized geometric model of the inserted tools is shown in Fig. 3, which are presented starting with the placement of the tool tips on the cutter bodies, the identification of oblique tool angles needed in the cutting mechanics model and the kinematics of cutting operations. The geometry of the tool tips are defined in their local coordinate system $O_{T}$ analytically. The control points are derived as functions of insert parameters that have been adapted from Kaymakci et al. (2012).

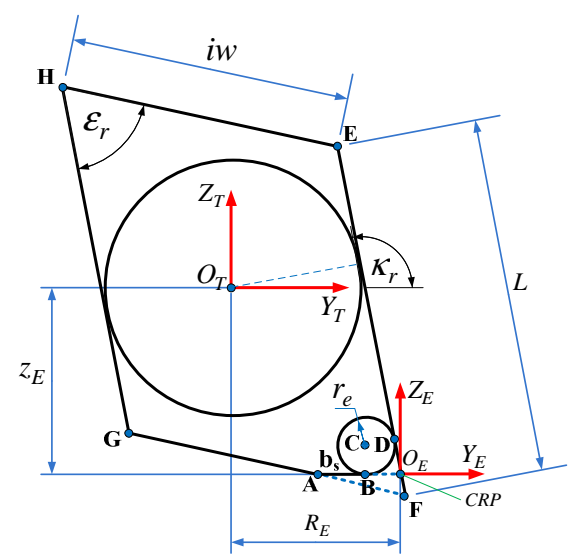

Fig. 3 Tool tips and cutting edges

The kinematic motions of the cutting edge against the reference coordinate system $O_{R}$ are presented by Eq. (4) including the kinematic deviations. 
Thasana, Sugimura, Iwamura and Tanimizu,

$$
\begin{aligned}
P_{x}= & \delta_{x}(C)-R_{T} \sin \theta_{z}+\varepsilon_{y}\left(\frac{i w \sin \left(\varepsilon_{r}+\kappa_{r}\right)}{2}+\frac{L \sin \kappa_{r}}{2}-\frac{\sin \left(\varepsilon_{r}-\kappa_{r}\right)\left(b_{s} \sin \kappa_{r}-r_{e} \cos \left(\kappa_{r}-1\right)\right)}{\sin \varepsilon_{r}}\right)-\sin \theta_{z}\left(\frac{i w \cos \left(\varepsilon_{r}+\kappa_{r}\right)}{2} \ldots .\right. \\
& \left.-b_{s}+\frac{L \cos \kappa_{r}}{2}+r_{e}\left(\cot \kappa_{r}-\frac{1}{\sin \kappa_{r}}\right)-\frac{\cos \left(\varepsilon_{r}+\kappa_{r}\right)\left(b_{s} \sin \kappa_{r}-r_{e} \cos \left(\kappa_{r}-1\right)\right)}{\sin \varepsilon_{r}}\right)-\varepsilon_{y} z_{B} \\
P_{y}= & \delta_{y}(C)+R_{T} \cos \theta_{z}-\varepsilon_{x}\left(\frac{i w \sin \left(\varepsilon_{r}+\kappa_{r}\right)}{2}+\frac{L \sin \kappa_{r}}{2}-\frac{\sin \left(\varepsilon_{r}-\kappa_{r}\right)\left(b_{s} \sin \kappa_{r}-r_{e} \cos \left(\kappa_{r}-1\right)\right)}{\sin \varepsilon_{r}}\right)+\cos \theta_{z}\left(\frac{i w \cos \left(\varepsilon_{r}+\kappa_{r}\right)}{2} \ldots\right. \\
& \left.-b_{s}+\frac{L \cos \kappa_{r}}{2}+r_{e}\left(\cot \kappa_{r}-\frac{1}{\sin \kappa_{r}}\right)-\frac{\cos \left(\varepsilon_{r}+\kappa_{r}\right)\left(b_{s} \sin \kappa_{r}-r_{e} \cos \left(\kappa_{r}-1\right)\right)}{2 \sin \varepsilon_{r}}\right)+\varepsilon_{x} z_{B} \\
P_{z}= & \varepsilon_{x} \cos \theta_{z}+\varepsilon_{y} \sin \theta_{z}\left(\frac{i w \cos \left(\varepsilon_{r}+\kappa_{r}\right)}{2}-b_{s}+\frac{L \cos \kappa_{r}}{2}+r_{e}\left(\cot \kappa_{r}-\frac{1}{\sin \kappa_{r}}\right)-\frac{\cos \left(\varepsilon_{r}+\kappa_{r}\right)\left(b_{s} \sin \kappa_{r}-r_{e} \cos \left(\kappa_{r}-1\right)\right)}{\sin \varepsilon_{r}}\right. \\
& -z_{B}+\frac{i w \sin \left(\varepsilon_{r}+\kappa_{r}\right)}{2}+\frac{L \sin \kappa_{r}}{2}+R_{T}\left(\varepsilon_{x} \cos \theta_{z}+\varepsilon_{y} \sin \theta_{z}\right)-\frac{\sin \left(\varepsilon_{r}-\kappa_{r}\right)\left(b_{s} \sin \kappa_{r}-r_{e} \cos \left(\kappa_{r}-1\right)\right.}{\sin \varepsilon_{r}}
\end{aligned}
$$

where,

$\begin{array}{ll}\varepsilon_{r} & : \text { Tool included (nose) angle } \\ i w & : \text { Insert width } \\ L & : \text { Insert length } \\ \kappa_{r} & : \text { Cutting edge angle of the insert } \\ b_{s} & : \text { Wiper edge length } \\ r_{e} & : \text { Corner radius }\end{array}$

\subsection{Generation of 3-dimensional bored face}

The simulation of the boring processes is carried out and a set of points are analytically generated to represent the machined faces including the kinematic deviations. Figure 4 illustrates the procedure of the virtual boring processes based on the input data of the kinematic deviations, the cutting conditions, the inserted tool tip parameters. In the figure, $f, v_{c}$ and $t_{w}$ are feed per tooth, cutting speeds and lengths of bored holes, respectively.

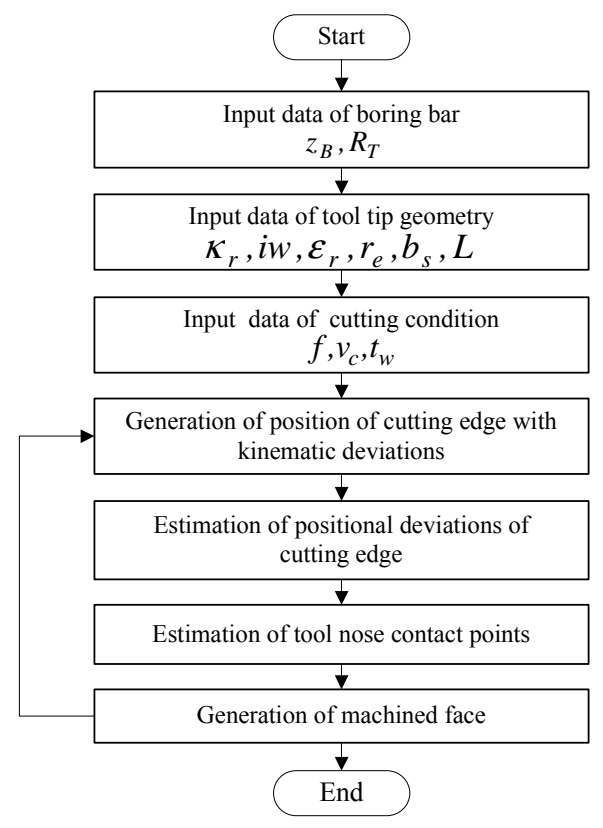

Fig. 4 Flow chart for boring process simulation

Once the position of the cutting edge is obtained, the next step is to generate the all points on the generated face of 
the workpiece. The cutting edge of a single point cutting tool has a nose radius, therefore, the contact points of the cutting edge and the workpiece can be described in the cross sectional plane represented by YZ-plane shown in Fig. 5. The critical tool angles are estimated for representing the extent of the tool nose, which generate the machined face. When the kinematic deviations are considered, the tool nose centers are no longer on a straight line, and the extents of the tool nose also changes continuously. Under these conditions, the discretized tool nose radius can then be represented and evaluated.

In the Fig. 5, $C_{i-1}, C_{i}$ and $C_{i+1}$ are the tool nose center locations at three consecutive revolutions of the spindle, $r_{e i-1}$, $r_{i}$ and $r_{e i+1}$ are the corresponding tool nose radius, and $P_{i n t+1}$ is the intersection point and the tool contact angle $\phi_{i j}$. Based on the locations of the tool nose centers for three consecutive revolutions at the same angular position of the spindle, based on the contact angle $\phi_{i j}$ of the tool nose during the second revolution can be calculated and the tool nose can be discretized.

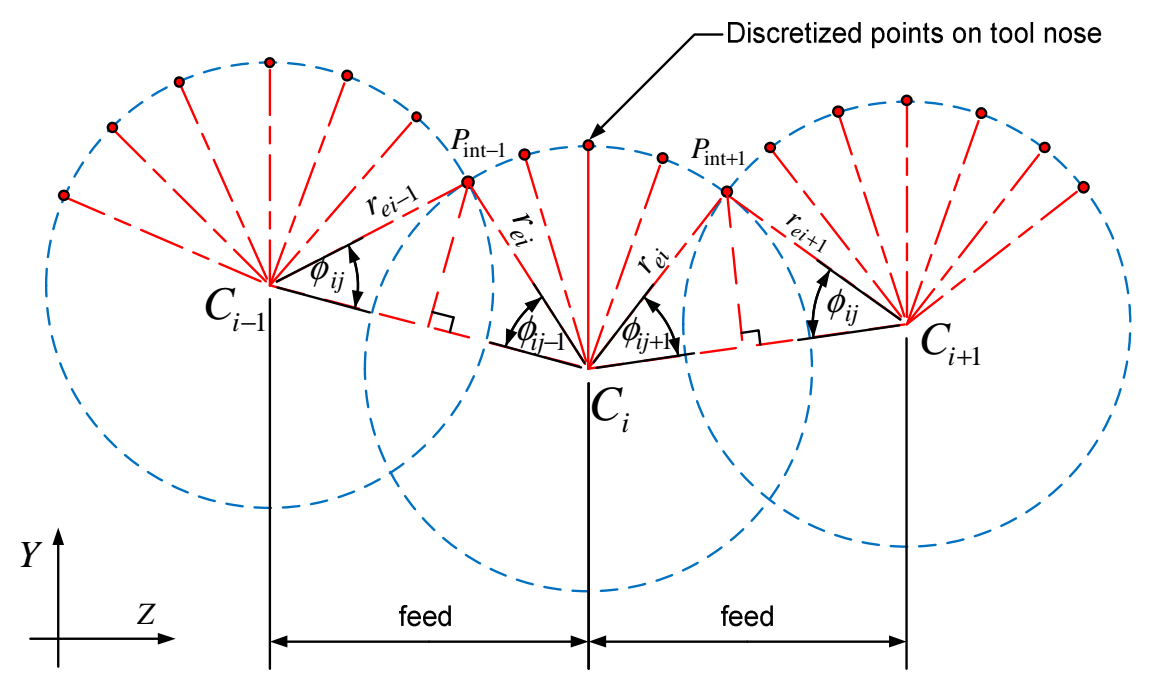

Fig. 5 Determination of groove size formed by tool nose geometry with kinematic deviations

The start and end contact angles of the tool nose with the workpiece can be calculated by the following equation in triangle $P_{i n t-1}, C_{i-1}$, and $C_{i}$ that have been adapted from Ramaswami (2010).

$\phi_{i j-1}=\cos ^{-1}\left[\frac{\overrightarrow{P_{\mathrm{int}-1} C_{i}} \bullet \overrightarrow{C_{i-1} C_{i}}}{\left|\overrightarrow{P_{\mathrm{int}-1} C_{i}}\right|\left|\overrightarrow{C_{i-1} C_{i}}\right|}\right]$

In triangle $\mathrm{P}_{i n t+1}, C_{i+1}$, and $C_{i}$, Eq. (6) is obtained.

$\phi_{i j+1}=\cos ^{-1}\left[\frac{\overrightarrow{P_{\mathrm{int}+1} C_{i}} \cdot \overrightarrow{C_{i+1} C_{i}}}{\left|\overrightarrow{P_{\mathrm{int}+1} C_{i}}\right|\left|\overrightarrow{C_{i+1} C_{i}}\right|}\right]$

At any given tool nose locations related to the angular orientation ' $\theta_{z}$ ' of the spindle with respect to the reference orientation, the span of the tool contact angle between the start and end angle is can be divided as required. The profile of the machined face can then be generated as a function of the final position of the cutting edges considering all kinematic deviations, by applying the following equation. 


$$
\begin{aligned}
& x=E_{x} \\
& y=R_{T}+P_{i n t+i}\left(\cos \phi_{i j}\right) \\
& z=P_{i n t+i}\left(\sin \phi_{i j}\right)
\end{aligned}
$$

where; $\mathrm{x}, y, \mathrm{z}$ is the position of a point of the tool nose, $R_{T}$ is the radial position of tool tip, and $E_{x}$ is the geometric deviations of position in the $\mathrm{X}$-axis.

The transformation from the YZ-plane to the appropriate orientation depending on the spindle angular orientation can be performed using the following transformation matrix.

$T=\left[\begin{array}{cccc}\cos \theta_{z} & -\sin \theta_{z} & 0 & 0 \\ \sin \theta_{z} & \cos \theta_{z} & 0 & 0 \\ 0 & 0 & 1 & F_{t} \\ 0 & 0 & 0 & 1\end{array}\right]$

$\left[\begin{array}{c}X_{\text {surface }} \\ Y_{\text {surface }} \\ Z_{\text {surface }} \\ 1\end{array}\right]=T\left[\begin{array}{c}x \\ y \\ z \\ 1\end{array}\right]$

$F_{t}=(f) \cdot\left(\frac{\theta_{z}}{2 \pi}\right)$

where; $X_{\text {surface }}, Y_{\text {surface }}, Z_{\text {surface }}$ represent the positions of a point on the machined face given by the point with $x, y$ and $z$, and $F_{t}$ is the feed rate per revolution

\section{Verification of 3-Dimensional Surface Roughness}

\subsection{Generation of reference datum}

Measurement of form deviations of engineering components are carried out with respect to a reference datum or trajectory. The deviations have to be measured against to the nominal geometric elements. ISO specifies that the actual measurement such that maximum deviation between the datum and the actual features concerned is the least squares for evaluating the form errors (Shunmugam, 1987). Assessment cylinders shown in Fig. 6 are considered here as the reference datum to measure the 3-dimensional surface roughness of the bored surfaces. The assessment cylinders are generated based on all the 3-dimensional coordinate data generated by the 3-dimensional boring process simulations.

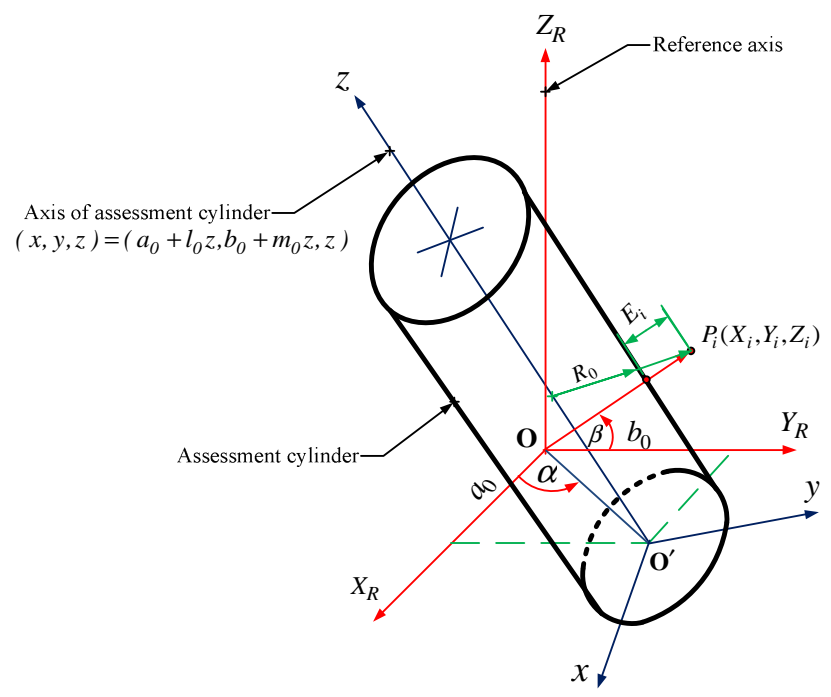

Fig. 6 Coordinate system for measuring deviations 
A procedure is proposed to generate an assessment cylinder in order to verify the 3-dimensional surface roughness based on all the trajectories of the cutting edge in the boring process. The parameters to be determined here are summarized in the following, which represent a cylinder in the reference coordinate system $X_{R} Y_{R} Z_{R}$.

(1) Unit vector $\left(l_{0}, m_{0}, n_{0}\right)$ representing the direction of the axis of the assessment cylinder,

(2) Vector $\left(a_{0}, b_{0}, 0\right)$ representing the position of the axis of the assessment cylinder, and

(3) $R_{0}$ representing the radius of the assessment cylinder.

The reference coordinate system $X_{R} Y_{R} Z_{R}$ coincides the one in Fig. 2, therefore, the parameters $l_{0}$ and $m_{0}$ are fixed, the parameters $a_{0}, b_{0}$, and $R_{0}$ are estimated by applying the following equations, based on the least mean square method (Thomas and Chan, 1989).

$a_{0}=\frac{c_{1} b_{2}-c_{2} b_{1}}{a_{1} b_{2}-a_{2} b_{1}}$

$b_{0}=\frac{a_{1} c_{2}-a_{2} c_{1}}{a_{1} b_{2}-a_{2} b_{1}}$

$R_{0}=\frac{1}{N}\left\{\sum_{i=1}^{N} X_{i}{ }^{2}-2 \sum_{i=1}^{N} X_{i} a_{0}+N a_{0}{ }^{2}+\sum_{i=1}^{N} Y_{i}^{2}-2 \sum_{i=1}^{N} Y_{i} b_{0}+N b_{0}{ }^{2}\right\}$

where,

$$
\begin{aligned}
& a_{1}=2\left(\left(\sum_{i=1}^{N} X_{i}\right)^{2}-N \sum_{i=1}^{N} X_{i}^{2}\right), \quad b_{1}=2\left(\sum_{i=1}^{N} X_{i} \sum_{i=1}^{N} Y_{i}-N \sum_{i=1}^{N} X_{i} Y_{i}\right) \\
& a_{2}=2\left(\sum_{i=1}^{N} X_{i} \sum_{i=1}^{N} Y_{i}-N \sum_{i=1}^{N} X_{i} Y_{i}\right), \quad b_{2}=2\left(\left(\sum_{i=1}^{N} Y_{i}\right)^{2}-N \sum_{i=1}^{N} Y_{i}^{2}\right) \\
& c_{1}=\left(\sum_{i=1}^{N} X_{i}^{2} \sum_{i=1}^{N} X_{i}-N \sum_{i=1}^{N} X_{i}^{3}+\sum_{i=1}^{N} X_{i} \sum_{i=1}^{N} Y_{i}^{2}-N \sum_{i=1}^{N} X_{i} Y_{i}^{2}\right) \\
& c_{2}=\left(\sum_{i=1}^{N} X_{i}^{2} \sum_{i=1}^{N} Y_{i}-N \sum_{i=1}^{N} Y_{i}^{3}+\sum_{i=1}^{N} Y_{i} \sum_{i=1}^{N} Y_{i}^{2}-N \sum_{i=1}^{N} X_{i}^{2} Y_{i}\right)
\end{aligned}
$$

The least mean square errors $E_{i}$ of all the generated point $P_{i}\left(X_{i}, Y_{i}, Z_{i}\right)$ and the assessment cylinder is given in the following equation, as shown in Fig. 6.

$$
E_{i}=\left(\left(X_{i}-l_{0} Z_{i}-a_{0}\right)^{2}+\left(Y_{i}-m_{0} Z_{i}-b_{0}\right)^{2}\right)^{1 / 2}-R_{0}
$$

The sum of the squares of the deviation is as follows.

$$
E_{s}=\sum_{i=1}^{N} E_{i}^{2}
$$

Therefore, the parameters of the assessment cylinders are estimated by applying the following procedure. STEP 1: Setting of parameters $l_{0}$ and $m_{0}$ 
The parameters $l_{0}$ and $m_{0}$ are set in the initial values -0.010 and moved to +0.010 by the step of 0.001 . STEP 2: Estimation of parameters $a_{0}, b_{0}$, and $R_{0}$

The parameters $a_{0}, b_{0}$, and $R_{0}$ are estimated by applying the Eqs. (10) to (12) for all the combination of $l_{0}$ and $m_{0}$. STEP 3: Estimation of least mean square errors $E_{s}$ and select an optimal parameters.

The least mean square errors $E_{s}$ is calculated by applying Eq. (14) based on the parameters of $l_{0}, m_{0}, a_{0}, b_{0}$ and $R_{0}$ and the generated points $P_{i}\left(X_{i}, Y_{i}, Z_{i}\right)$. A combination of the parameter values which minimize $E_{s}$ is selected as the parameters representing the assessment cylinder. The parameters $l_{0}, m_{0}, a_{0}, b_{0}$ and $R_{0}$ obtained here give the position and orientation errors and the dimensional error in the radius of the bored cylinders generated by the simulations.

\subsection{Estimation of surface roughness}

After generating the assessment cylinders, the 2-dimensional (2D) surface roughness $R_{\max }, R_{a}$ and $R_{q}$ defined in ISO 4287:1997 are estimated by Eqs. (15) to (17). On the other hand, the 3-dimensional (3D) surface roughness has not yet been applied widely. Therefore, a capital letter " $S$ " (for Surface) is used to identify 3D parameters, in order to distinguish 2D and 3D parameters.

The 3D surface roughness was proposed (Dong et al. 1994; Quinsat et al. 2008; Abouelatta, 2010) to assess the 3D surface topography base on the least squares mean plans. The 3D surface roughness parameter of $S_{\max }$ has been estimated similar with the $2 \mathrm{D}$ surface roughness, but 3D surface roughness is evaluated from all the points of the generated faces. The 3D surface roughness parameters of $S_{a}$ and $S_{q}$ are the arithmetic mean deviation of the assessed profile and the root mean square deviations of the assessed profile that have been adapted from Abouelata (Abouelatta, 2010), Dong et al. (Dong et al. 1994) and Quinsat et al. (Quinsat et al. 2008). The 2D and 3D surface roughness parameters are estimated by applying the following equations.

$R_{\max }=r_{e}-\max \left\{\left|E_{i}\right|\right\}$

$R_{a}=\frac{1}{L} \int_{x=0}^{x=L}|y| d x \cong \frac{1}{n} \sum_{i=1}^{n}\left|E_{i}\right|$

$R_{q}=\left(\frac{1}{L} \int_{x=0}^{x=L} y^{2} d x\right)^{1 / 2} \cong\left(\frac{1}{n} \sum_{i=1}^{n}\left(E_{i}\right)^{2}\right)^{1 / 2}$

$S_{\max }=r_{e}-\max \left\{\left|E_{i}\right|\right\}$

$S_{a}=\frac{1}{N} \sum_{i=1}^{N}\left|E_{i}\right|$

$S_{q}=\sqrt{\frac{1}{N} \sum_{i=1}^{N}\left(E_{i}\right)^{2}}$

where,

$R_{\max } \quad$ : Maximum height profile of 2-Dimensional surface roughness

$R_{a} \quad:$ Arithmetic mean deviation of 2-Dimensional surface roughness

$R_{q} \quad:$ Root mean square deviation of 2-Dimensional surface roughness

$S_{\max } \quad$ : Maximum height profile of 3-Dimensional surface roughness

$S_{a} \quad$ : Arithmetic mean deviation of 3-Dimensional surface roughness

$S_{q} \quad$ : Root mean square deviation of 3-Dimensional surface roughness 


\section{Case Studies}

\subsection{Boring process simulation}

Figure 7 show examples of the generated faces by the boring simulation considering kinematic deviations. As shown in the figure, a set of the points on the machined faces are generated. The geometric deviations of any points on the machined faces can be evaluated by comparing the generated faces with the kinematic deviations and one without any deviations.

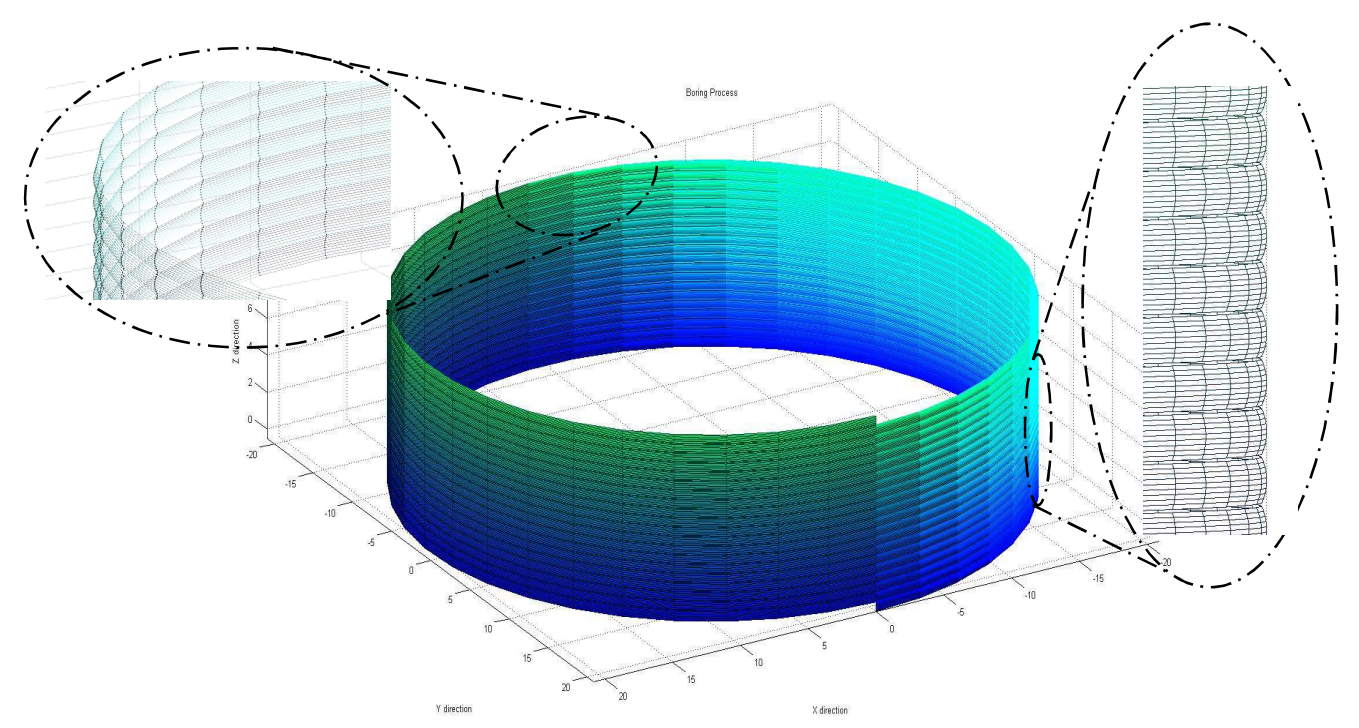

(a) Feed rate: $0.5 \mathrm{~mm} / \mathrm{rev} ; z_{B}: 73 \mathrm{~mm} ; R_{T}: 20 \mathrm{~mm} ; r_{e}: 0.8 \mathrm{~mm} ; \kappa_{r}: 100^{\circ} ; \varepsilon_{r}: 80^{\circ} ; i w: 6.35 \mathrm{~mm} ; b_{s}: 0 \mathrm{~mm}$; $L: 6.35 \mathrm{~mm}$; Positioning and orientation deviations $=0$

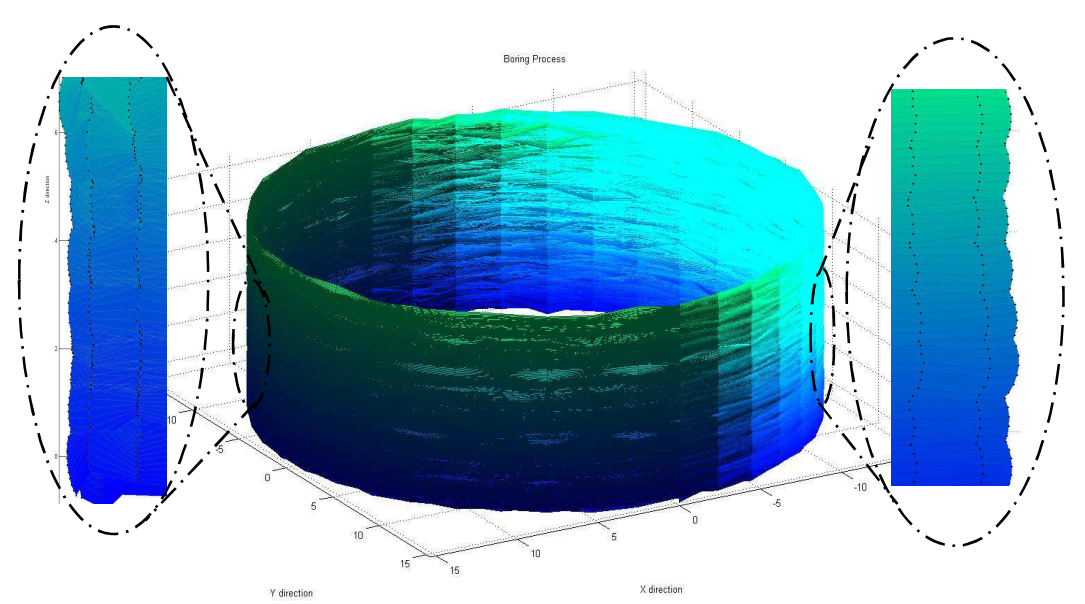

(b) Feed rate: $0.5 \mathrm{~mm} / \mathrm{rev} ; z_{B}: 73 \mathrm{~mm} ; R_{T}: 15 \mathrm{~mm} ; r_{e}: 0.8 \mathrm{~mm} ; \kappa_{r}: 100^{\circ} ; \varepsilon_{r}: 80^{\circ} ; i w: 6.35 \mathrm{~mm} ; b_{s}: 0 \mathrm{~mm}$; $L: 6.35 \mathrm{~mm}$; Position deviations are given randomly, normal distribution $\mathrm{N}(0,5 \mu \mathrm{m})$; Orientation deviations are given randomly, normal distribution $\mathrm{N}(0,10 \mu \mathrm{rad})$

Fig. 7 Estimated geometries of bored faces considering kinematic motion deviations

\subsection{Estimation of 3-dimensional surface roughness and discussions}

All the points on the bored faces are obtained through the simulation, as shown in Fig. 7. The surface roughness of the generated faces are summarized in the tables 1 (a) and (b) based on the simulation results under four different machining parameters of the feed rates and corner radius of inserted tool tips. 
Table 1 (a) shows the comparisons between the theoretical values of the 2-dimensional (2D) surface roughness calculated from the feed rates and the nose radius, and the 2D and the 3D surface roughness obtained through the simulations. As regards the $2 \mathrm{D}$ surface roughness, the estimated values of $R_{a}$ and $R_{\max }$ are almost same as the theoretical values $R_{a_{-} t h}$ and $R_{\text {max_t } t}$. The estimated values are obtained from 40,000 points generated by the boring process simulation.

The percentage of maximum differences between the results of the theoretical and estimated values is less than $0.90 \%$ for $R_{a}$ and $2.33 \%$ for $R_{\max }$. The 3D surface roughness $S_{a}$ and $S_{\max }$ are smaller than the 2-dimensional ones.

Table 1 (b) shows the simulation results of the 2D and 3D surface roughness obtained by the boring processes with kinematic deviations. The values of $3 \mathrm{D}$ surface roughness generally give smaller values than $2 \mathrm{D}$ surface roughness in most cases. The smallest value of the surface roughness is obtained by the machining conditions of $f=0.05 \mathrm{~mm} / \mathrm{rev}$ and $r_{e}=0.2 \mathrm{~mm}$ for using the analysis conditions shown in the followings.

(1) Position deviations are given randomly as a normal distribution $\mathrm{N}(0,5 \mu \mathrm{m})$, and

(2) Orientation deviations are given randomly as a normal distribution $\mathrm{N}(0,10 \mu \mathrm{rad})$.

Table 1 Estimated surface roughness

(a) Surface roughness without kinematic deviations

\begin{tabular}{|c|c|c|c|c|c|c|c|c|c|c|c|c|c|c|}
\hline \multirow{4}{*}{$\begin{array}{c}\text { Test } \\
\text { no. }\end{array}$} & \multicolumn{2}{|c|}{$\begin{array}{c}\text { Cutting } \\
\text { Condition }\end{array}$} & \multicolumn{2}{|c|}{ Theoretical } & \multicolumn{10}{|c|}{ Surface Roughness of Simulations } \\
\hline & \multirow{3}{*}{$\begin{array}{c}f \\
{[\mathrm{~mm}} \\
/ \mathrm{rev}]\end{array}$} & \multirow{3}{*}{$\begin{array}{c}r_{e} \\
{[\mathrm{~mm}]}\end{array}$} & \multirow{3}{*}{$\begin{array}{c}R_{\text {max_th }} \\
{[\mu \mathrm{m}]} \\
\text { (a) }\end{array}$} & \multirow{3}{*}{$\begin{array}{c}R_{a \_t h} \\
{[\mu \mathrm{m}]} \\
\text { (b) }\end{array}$} & \multicolumn{2}{|c|}{$\begin{array}{l}R_{\max } \\
{[\mu \mathrm{m}]}\end{array}$} & \multicolumn{2}{|c|}{$\begin{array}{c}R_{a} \\
{[\mu \mathrm{m}]}\end{array}$} & \multirow{2}{*}{$\begin{array}{c}\begin{array}{c}R_{q} \\
{[\mu \mathrm{m}]}\end{array} \\
\text { Predict }\end{array}$} & \multicolumn{2}{|c|}{$\begin{array}{l}S_{\max } \\
{[\mu \mathrm{m}]}\end{array}$} & \multicolumn{2}{|c|}{$\begin{array}{c}S_{a} \\
{[\mu \mathrm{m}]}\end{array}$} & \multirow{2}{*}{$\begin{array}{c}\begin{array}{c}S_{q} \\
{[\mu \mathrm{m}]}\end{array} \\
\text { Predict }\end{array}$} \\
\hline & & & & & Predict & $\begin{array}{l}\text { Diff. } \\
(\%)\end{array}$ & Predict & $\begin{array}{l}\text { Diff. } \\
(\%)\end{array}$ & & Predict & $\begin{array}{l}\text { Diff. } \\
(\%)\end{array}$ & Predict & $\begin{array}{l}\text { Diff. } \\
(\%)\end{array}$ & \\
\hline & & & & & (d) & (a)-(d) & (f) & (b)-(f) & $(\mathrm{g})$ & (h) & (a)-(h) & (i) & (b)-(i) & (j) \\
\hline 1 & 0.05 & 0.2 & 1.56 & 0.40 & 1.56 & 0.39 & 0.40 & -0.22 & 0.59 & 1.56 & 0.39 & 0.27 & 32.15 & 0.31 \\
\hline 2 & 0.1 & 0.4 & 3.13 & 0.80 & 3.11 & 0.39 & 0.80 & -0.24 & 1.19 & 3.11 & 0.39 & 0.54 & 32.12 & 0.63 \\
\hline 3 & 0.25 & 0.8 & 9.77 & 2.51 & 9.71 & 0.60 & 2.51 & -0.04 & 3.70 & 9.71 & 0.60 & 1.70 & 32.26 & 1.95 \\
\hline 4 & 0.5 & 0.8 & 39.06 & 10.03 & 38.15 & 2.33 & 9.94 & 0.90 & 14.67 & 38.15 & 2.33 & 6.73 & 32.90 & 7.75 \\
\hline
\end{tabular}

(b) Surface roughness with kinematic deviations

\begin{tabular}{|c|c|c|c|c|c|c|c|c|c|}
\hline \multirow{2}{*}{$\begin{array}{c}\text { Test } \\
\text { no. }\end{array}$} & \multicolumn{3}{|c|}{ Cutting Condition } & \multicolumn{6}{|c|}{ Surface Roughness of Simulations } \\
\cline { 2 - 9 } & $\begin{array}{c}R_{T} \\
{[\mathrm{~mm}]}\end{array}$ & $\begin{array}{c}f \\
{[\mathrm{~mm} / \mathrm{rev}]}\end{array}$ & $\begin{array}{c}r_{e} \\
{[\mathrm{~mm}]}\end{array}$ & $\begin{array}{c}R_{\max } \\
{[\mu \mathrm{m}]}\end{array}$ & $\begin{array}{c}R_{a} \\
{[\mu \mathrm{m}]}\end{array}$ & $\begin{array}{c}R_{q} \\
{[\mu \mathrm{m}]}\end{array}$ & $\begin{array}{c}S_{\max } \\
{[\mu \mathrm{m}]}\end{array}$ & $\begin{array}{c}S_{a} \\
{[\mu \mathrm{m}]}\end{array}$ & $\begin{array}{c}S_{q} \\
{[\mu \mathrm{m}]}\end{array}$ \\
\hline 1 & 15 & 0.05 & 0.2 & 27.63 & 8.91 & 13.21 & 27.63 & 9.20 & 10.78 \\
2 & 15 & 0.1 & 0.4 & 35.77 & 9.55 & 5.44 & 35.77 & 9.36 & 10.95 \\
3 & 15 & 0.25 & 0.8 & 45.99 & 9.94 & 15.85 & 45.99 & 9.99 & 11.97 \\
4 & 15 & 0.5 & 0.8 & 60.17 & 13.25 & 16.88 & 60.17 & 9.56 & 11.82 \\
\hline
\end{tabular}

\section{Conclusions}

A systematic method is proposed here to estimate both the two-dimensional (2D) and three-dimensional (3D) surface roughness through the boring process simulations with kinematic motion deviations. The followings are concluded.

(1) A model is proposed to represent the kinematic motions of the cutting edges against the reference coordinate systems of the spindle heads, taking into consideration of the kinematic deviations of the boring tool systems.

(2) A systematic method is proposed to estimate the geometric deviations of the machined face based on the kinematic motions of the cutting edges. 
(3) A proposed model and method are applied to the simulation of the simple boring process, and the geometries of the machined faces are estimated, based on the cutting conditions, the tool geometries and the kinematic deviations of the boring processes.

(4) A method is also proposed to estimate both the $2 \mathrm{D}$ and $3 \mathrm{D}$ surface roughness based on the boring process simulation with the kinematic motion deviations. The proposed method provides us with a systematic method to verify and to evaluate the surface roughness based on the least mean square cylinders.

\section{References}

Abdul, K. A., Xu, X. and Hammerle, E., Virtual machine tools and virtual machining - A technological review, Robot. Comp. - Integr. Manuf., Vol.27 (2011), pp.494-508.

Abouelatta, O. B., 3D surface roughness measurement using a light sectioning vision system, Proc. of the World Congress on Engineering 2010, IAENG (online), available from < http://www.iaeng.org/publication/WCE2010 /WCE2010_pp698-703.pdf>, (accessed on 2 July, 2010).

Atabey, F., Lazoglu, I. and Altintas, Y., Mechanics of boring processes-Part I, Int. J. Mach. Tools Manuf., Vol.43 (2003), pp.463-476.

Buj-Corral, I., Vivancos-Calvet, J. and Dominquez-Fernandez, A., A surface topography in ball-end milling process as a function of feed per tooth and radial depth of cut, Int. J. Mach. Tools Manuf., Vol.53 (2012), pp.151-159.

Choi, J. P., Lee, S. J. and Kwon, H. D., Roundness error prediction with a volumetric error model including spindle error motions of a machine tool, Int. J. Adv. Manuf. Technol., Vol.21 (2003), pp.923-928.

Dong, W. P., Sullivan, P. J. and Stout, K. J., Comprehensive study of parameters for characterizing three dimensional surface topography III: Parameters for characterizing amplitude and some functional properties, Wear, Vol.178 (1994), pp.29-43.

Govik, A., Nilsson, L. and Moshfegh R., Finite element simulation of the manufacturing process chain of a sheet metal assembly, J. Mater. Proc. Technol., Vol.212 (2012), pp.1453-1462.

ISO 230-1: 2012, Test code for machine tools -Part 1: Geometric accuracy of machines operating under no-load or quasi-static conditions, ISO (2012), pp.107-109.

Kaymakci, M., Kilic, Z. M. and Altintas, Y., Unified cutting force model for turning, boring, drilling and milling operations, Int. J. Mach. Tools Manuf., Vol.54-55 (2012), pp.34-45.

Li, J. G., Zhao, H., Yao, Y. X. and Liu, C. Q., Off-line optimization on NC machining based on virtual machining, Int. J. Adv. Manuf. Technol., Vol.36 (2008), pp.908-917.

Luo, L., Li, G., Sun, S. and Meng Q., Research on behavior simulation of multi-axis CNC machine tool in virtual environment, Int. Conf. on Measuring Technology and Mechatronics Automation (2010), pp.31-34.

Quinsat, Y., Sabourin, L. and Lartigue, C., Surface topography in ball end milling process: Description of a 3D surface roughness parameter, J. Mater. Proc. Technol., Vol.95 (2008), pp.35-43.

Ramaswami, H., An integrated framework for virtual machining and inspection of turned parts, Ph.D. Thesis, University of Cincinnati, pp.41-45, OhioLINK (online), available from <http://rave.ohiolink.edu/etdc/view?acc_nu $\mathrm{m}=\mathrm{ucin} 1282574694>$, (accessed on 5 April, 2010).

Shunmugam, M. S., Comparison of linear and normal deviations of forms of engineering surfaces, Precision Engineering, Vol.9, No.2 (1987), pp.96-102.

Shaw, M. C., Metal Cutting Principles $2^{\text {nd }}$ ed. (2005), Oxford University Press.

Sugimura, N., Watabiki, H., Thasana, W., Iwamura K. and Tanimizu, Y., Analysis of kinematic motion deviations of rotary tables based on geometric tolerances, J. Adv. Mech. Design Sys. Manuf., Vol.6, No.7 (2012), pp.1132-1142.

Sugimura, N. and Murabe, A., A study an analysis of alignment errors of 5-axis machine tools, Proc. of Int. Conf. on Manufacturing Milestones toward the $27^{\text {st }}$ Century, JSME (1997).

Thomas, S. M. and Chan, Y. T., A simple approach for the estimation of circular arc center and its radius, Comp. Vis. Graph. Im. Proc., Vol.45 (1989), pp.362-370. 